\title{
Germanica
}

GERMANICA $49 \mid 2011$

Péripéties du snobisme

\section{Franz Werfel. Le snobisme, père de tous les maux}

\section{Michel Reffet}

\section{(2) OpenEdition}

\section{Journals}

Édition électronique

URL : http://journals.openedition.org/germanica/1371

DOI : 10.4000/germanica. 1371

ISSN : 2107-0784

\section{Éditeur}

Université de Lille

\section{Édition imprimée}

Date de publication : 31 décembre 2011

Pagination : 69-82

ISBN : 9782913857285

ISSN : 0984-2632

\section{Référence électronique}

Michel Reffet, «Franz Werfel. Le snobisme, père de tous les maux », Germanica [En ligne], 49 | 2011, mis en ligne le 23 mars 2012, consulté le 06 octobre 2020. URL : http://journals.openedition.org/ germanica/1371 ; DOI : https://doi.org/10.4000/germanica.1371

Ce document a été généré automatiquement le 6 octobre 2020.

(c) Tous droits réservés 


\title{
Franz Werfel. Le snobisme, père de tous les maux
}

\author{
Michel Reffet
}

Dans une acception courante, être snob semble bien innocent. La tradition lexicographique définit le snobisme comme l'effort de se conformer à tout ce qui est en vogue : vêtement, sources de l'information et de la culture, fréquentation de certains lieux de réunion. Plus précisément, le snob veut se distinguer de la masse - démarche individualiste - et adhérer à une mode - démarche conformiste. Le milieu qu'il vise est privilégié puisque libre de choisir un genre de vie original, mais créatif et lucratif, sécurisé par l'assentiment du groupe et la tolérance sociétale.

2 Comment se fait-il donc qu'un auteur en vue, en son temps nommé à maintes reprises, à côté de Thomas Mann, comme la figure la plus éminente de la littérature de langue allemande, présente le snobisme comme " une force intellectuelle universelle »? Der Snobismus als geistige Weltmacht ${ }^{1}$ - tel est le titre d'un de ses grands essais, paru en 1928.

3 Selon Werfel le snobisme est le dénominateur commun des manifestations collectives des années 20 les plus pernicieuses, diffusées par la presse «jusque dans les vaisseaux capillaires du corps social » (« ... in alle Haaräderchen des Gesellschaftskörpers » 271) : volonté de se faire valoir par tous les moyens, révolutions arbitraires, défaitisme moral, obscurité de l'expression, imitation par renoncement à l'effort créatif, perversité (luxure, prostitution généralisée, mépris de la virginité, homosexualité), recours à la drogue, admiration pour l'énergie criminelle - «Das Verbrechen rückt zu einer Sportart auf $»^{2}(274)$-, ce qui est imputable à l'anglo-américanisme - « das herrschende englisch-amerikanische Ideal, ein kinetisch-rationalistisches Ideal, das noch dazu in unseren Ländern demoralisiert wird $»^{3}$; c'est-à-dire un modèle reposant sur le mouvement incessant ( kinetisch»), le sport, la boxe, véhiculé par le cinéma; ce modèle, il devient facile de le vider de toute morale («demoralisiert»), ce dont se chargent les imitateurs Européens (275).

Werfel renonce à une base d'investigation socio-historique du snobisme. Il avoue ne pas connaître le classique "Snobsbuch ${ }^{4}$, dont il ne nomme même pas l'auteur (262). Il mentionne comme en passant l'origine la plus couramment admise du mot snob, 
abréviation de sine nobilitate, notée sur la porte des chambres à la suite du nom des rares étudiants roturiers des Universités de Cambridge et Oxford (265) ; sans s'attarder sur cet usage discriminatoire, on passe tout droit au Bourgeois gentilhomme, « représentation classique du snob» (« Klassische Darstellung des Snobs ») (265).

Werfel avait annoncé d'emblée qu'il n'allait pas s'arrêter à une définition simple et péremptoire du snobisme. Elle se fait attendre, vu que de toute façon, il s'agit de la faire dévier de son sens originel et courant. Werfel commence par botter en touche vers Schnitzler, dont il reprend la phrase: "Snobismus ist [...] in jedem Menschen als potentieller Krankheitsherd zu finden $»^{5}$ (262). Puis Werfel creuse et affine cette idée de maladie, pour en arriver à sa propre assertion originale: "Snobismus ist der krankgewordene Wille zur Geltung $»^{6}(263)$. Cette définition parcourt dès lors l'essai dans son entier : "instinct maladif de se faire valoir ", «fantasme pathologique de sa propre valeur » («kranker Geltungstrieb», «kranker Geltungswahn »). Au regard de cette grave pathologie, la fébrilité du Bourgeois gentilhomme n'est pas ce qu'il y a de pire: "Der Aristokraten-, Kirchen-, Künstler-, Berühmtheit-Snob ist die harmloseste Wald- und Wiesenspezies " ${ }^{7}$ (267).

6 Il ne faut toutefois pas s'y tromper. Passé le niveau de la comédie, le refus de sa condition débouche sur des aliénations définitives : trahison de ses parents et origines; reniement de sa vraie patrie ; état de suicide permanent, puisque abandon de sa vraie nature (266). En littérature, Werfel s'en prend à Stendhal, qu'il identifie à son héros Julien Sorel; les admirateurs de Stendhal, dit-il, sont aussi des snobs! (269-270) Également snob est Nietzsche, puisque, reniant toute évidence, il se défend d'être allemand et insiste ingénument sur ses origines polonaises (270).

7 Les acceptions les plus banales du snobisme prennent des dimensions psychanalytiques, appuyées par un renvoi en note aux travaux de Alfred Adler sur cette pulsion maladive. La haine de soi produit un Torquemada, d'origine juive et massacreur de Juifs (267) ; les rêves de gloire produisent un Napoléon. Le radicalisme en politique relève lui aussi du snobisme. Le snob communiste, qui ignore tout du prolétariat, a pris le relais de l'arriviste d'autrefois, qui ne connaissait rien de l'aristocratie. Et les pires tenants du nationalisme sont souvent des étrangers en mal d'intégration (267-269).

Ich könnte, wenn ich irgendeine fanatische Blutrünstigkeit lese, darauf schwören, dass der Verfasser ein menschenscheuer Neurotiker mit Gymnasialbildung ist, der, ein missglückter Torquemada, alle Hände und Füße voll zu tun hat, die Spuren seiner wahren Natur zu verwischen. Die Partei- und Klassenfarbe ist für unsere Studie vollkommen gleichgültig. Denn weit zahlreicher als der kommunistische tritt allenthalben der nationalistische Snob auf. Auch er beweist durch jenen verdächtigen Radikalismus seine Illegitimität, seine Nichtdazugehörigkeit. ${ }^{8}$ (268)

Grande est la responsabilité des expériences " futuristes », puisqu'en plus des violences faites à la forme, elles impliquent toutes les dérives. Werfel cite deux strophes d'une ballade loufoque badinant sur le cas de Hans Apfelböck, assassin de ses parents - sans toutefois nommer l'auteur, qui est Bertolt Brecht (273-274). Et c'est le poète Maïakovski (1893-1930), avant-gardiste devenu révolutionnaire stalinien, qui encadre les réflexions de Werfel: l'essai débute par l'évocation du «Maïakovski sourit» («Majakowski lächelt »), œuvre de jeunesse du dandy que Werfel classe comme un "Oscar Wilde à la puissance deux " - «Über-Wilde » - et se termine sur une citation du même, passé chantre officiel du pouvoir bolchevique, qui est un appel à la déshumanisation et à l'assassinat gratuit : 
Deine Seele : Dampf, Preßluft, Elektrizität !

Den Almosenspendern, Nabelbeschauern

Die Axt über die Glatze tanzen lassen!

Erschlagen Erschlagen $!^{9}$ etc. (278)

il s'agit de faire converger et d'ouvrir sur des perspectives nouvelles un référentiel d'idées ou de faits connus de tous et ne donnant guère lieu, de prime abord, à des interprétations variées. Les argumentations centrales s'annoncent subrepticement, s'enflent progressivement, à la faveur de rapprochements inattendus. Des investigations psychologiques approfondies ramènent à des lieux communs, ceux-ci trouvant par là un fondement anthropologique. La gradation dans la définition du snobisme se fait par anticipations justifiées après coup. Les exemples se répartissent entre phénomènes sociaux et phénomènes littéraires. Les deux catégories s'enchevêtrent, mais le lecteur est pris, convaincu non par la progression du raisonnement, mais par le prestige des personnages et auteurs cités, et par le dédale des références culturelles émaillant les affirmations de l'auteur. On donne l'illusion de l'objectivité en interprétant des faits incontestables. Tels se présentent, du reste, les essais littéraires en général ; l'essai est « recours à une forme d'écriture libérée de tout impératif de forme ou de canon préalablement défini »; "L'écrivain “essayiste" est une sorte de Protée qui se meut en toute liberté par-delà les siècles, les continents et les traditions établies. $»^{10}$

11 Werfel a l'art de poursuivre son idée maîtresse sous une multiplicité de formulations brillantes, bien frappées, mais redondantes. Il ne conduit pas la démonstration de façon soigneuse, "topique », mais inspirée. Il s'excuse d'une rupture de raisonnement («Gedankensprung ») là où il n'y en a pas vraiment (275). En fait l'essai agit comme un poème. Or un poème est réussi, quand les ellipses et illogismes ne créent pas de surprise, ne font pas obstacle à l'envoûtement du "récepteur » - auditeur ou lecteur. Werfel opère par une série de contrastes entre réalités et fruits de l'imagination: Tatsache/Theater, Besitz/Gebärde, Position/Rolle, Machtstellung/Geltung, Held/ Schauspieler, die Sache/der Glanz, Realität/Insignien, Gesetz/Übertretung ${ }^{11}$. L'amoureux de la musique qu'est Werfel approche le phénomène social et politique par le biais de la création musicale : de l'opposition de base positiv/negativ, Symmetrie/ Asymmetrie, on glisse vers Konsonanz/Dissonanz et Tonalität/Atonalität : « Es gibt eine Atonalität des Geltungstriebes ! Es ist der Snobismus $»^{12}$.

12 Werfel ne prétend pas que le besoin de se mettre en valeur soit une maladie. Il définit le snobisme comme une maladie qui affecte ce besoin inné. Ce qui est naturel à tout être humain, dit-il, c'est la volonté de puissance, reconnue désormais par « la psychologie post-nietzschéenne » :

Der Wille zur Macht! Man lausche genau dem Klang dieses Begriffes. Niemand kann seine Wirklichkeit leugnen. Aber er tönt nicht wie ein einfacher Klang, sondern wie ein Akkord, in dem sich mehreres zusammenfindet ${ }^{13}$ (262).

13 Jusqu'ici, tout est en ordre. Werfel, grand lecteur de Nietzsche dans sa jeunesse, parle d'un « son », d'un « accord ». Immédiatement suit la question : 
... ist es wahr, daß der Mensch von der Tatsache der Macht träumt, von den Strapazen, sie zu erringen, von den Verantwortungen, Gefahren, Bitternissen, sich in ihr zu behaupten ? (262-263) ${ }^{14}$

plupart du temps, les grands essais expriment les positions d'auteurs assez notoires pour penser que leurs jugements ont du poids auprès de leurs contemporains. Der Snobismus... condense les convictions explicites ou implicites insérées dans l'œuvre fictionnelle de Werfel depuis les années vingt. Verdi. Roman der Oper (1923), première œuvre majeure de la période, comporte maint passage déconnecté de la perspective narrative, où les jugements ne sont plus ceux d'un personnage, mais évidemment ceux de l'auteur. Ludwig Rohner dans son ouvrage de référence sur l'essai, remarque d'ailleurs: "Werfels Verdi ist schon im (antithetisch durchgeführten) Thema essayistisch $»^{17}$. On y prend parti pour Verdi contre Wagner et on dénigre les admirateurs de ce dernier. Or ce sont les «desperados de l'esprit » : « [die] seelischen Desperados aller Nationen, [die] Uneinfachen und Romantischen, deren internationaler Treffort Bayreuth wurde $»^{18}$. Une tirade spécialement féroce contre le romantisme avait précédé, dès le deuxième chapitre ${ }^{19}$ et plus loin on appelle Wagner carrément «le romantique allemand $»^{20}$. Cette équivalence est longuement développée dans l'essai de 1942 Ein Bildnis Giuseppe Verdis :le romantique, écrit Werfel, faisait bon marché de la réalité : «Der Romantiker setzte sich über die Wirklichkeit hinweg »² (401).Et dans le journal de son premier voyage en Égypte, en 1925, en découvrant à Louxor les sculptures prétentieuses laissées par Alexandre le Grand, il identifie purement et simplement le snob avec le romantique.

...schon die Verkleidung, die Sucht, in einem historischen Kostüm zu paradieren, dieses Kokettieren mit einer großen Vorzeit beweist einen Snob und Romantiker, einen der ersten Snobs, die uns geschichtlich bekannt sind (723) ${ }^{22}$

Le souvenir de la période hellénistique d'Égypte et de Palestine joue un rôle important dans le drame de 1926 Paulus unter den Juden («Saint Paul parmi les Juifs»), qui se passe à Jérusalem sous Caligula (37-41). On y voit un jeune Hiérosolymite du nom de Matthias, qui plus est, fils du Grand Prêtre, fasciné par la Grèce, qui apprend le grec, s'habille à la 
grecque et fréquente les gymnases grecs. Ce n'est pas pour élargir son horizon intellectuel, ni par amour de l'autre, mais par haine de ses origines. Il admire Frisius, commandant de la garnison romaine, parce que celui-ci est un Germain qui a su rompre avec les siens. Dès le démarrage du drame, Matthias avoue : «Ich habe einen Abscheu vor unserem Blut. Weißt du, was ich mit uns täte, wenn ich Cäsar wäre?! ». Il ne poursuit pas, mais suggère seulement une abomination ("das Furchtbare") ${ }^{23}$. Il fait partie de ces Juifs assimilés ou convertis, que, dans son essai, Werfel classe, eux aussi, parmi les snobs :

Ebenso gibt es zu Hunderten Juden, wenn auch gerade keine Torquemadas, die einen seligen Schwächeanfall bekommen, wenn sie Weihrauch spüren, und einen unseligen Schwächeanfall, wenn sie als das erkannt werden, was sie sind... ${ }^{24}$ (267)

De leur reniement, il trouve des exemples vivants avec Otto Weininger et Karl Kraus. Dans son journal de juillet 1918, il note à la suite de la lecture des Letzte Dinge (Choses dernières) de Weininger : « Das ist der Leidenschaftszustand des Selbsthasses... » ${ }^{25}$ (645). Quant à Karl Kraus, on connaît sa conversion, son baptême et tout son mépris envers Juifs et sionistes. Dès 1917, après sa brouille avec Kraus, Werfel lui avait écrit dans une lettre ouverte : «Sie sind nicht nur der Sohn Ihres Volkes, sondern vor allem der Sohn der Assimilation, jenes dunklen Zwanges, alle Spur hinter sich verwischen oder persiflieren zu müssen $»^{26}(584)$. Dans son Journal de 1920, il avait imaginé un récit satirique qu'il intitula Le Parent (Der Verwandte), où le publiciste Karl Kalan, alors qu'il attendait la venue d'une femme de l'aristocratie, reçoit la visite impromptue de son cousin, reconnaissable comme Juif galicien. Dérangé dans son programme, Kalan empoisonne le visiteur importun et cache son cadavre dans son appartement. Il voulait à tout prix éviter que son amie s'aperçoive qu'il a de la parenté juive. La dame arrive, découvre le forfait, et l'on peut supposer qu'elle prend le large (667-670). On reconnaît sans peine le cas Karl Kraus, chroniqueur lié à une comtesse, Sidonie Nadherny von Borutin. Toutefois, la parabole sur le snob honteux d'être reconnu comme Juif resta à l'état de fragment et ne fut pas publiée, Werfel se refusant sans doute à polémiquer sur le plan personnel. D'ailleurs, mise à part une attaque contre Kraus insérée dans le drame Spiegelmensch (L'Homme miroir), également de 1920, et qui fut exclue de la représentation sur scène, Werfel abandonna la partie, allant de succès en succès ${ }^{27}$. Comme rappelé ci-dessus, Werfel croyait bon de préciser que tous les Juifs renégats ne sont pas des Torquemada! Or curieusement, Karl Kraus lui-même, dans un délire de purisme et de toute-puissance, se vantait d'être un Torquemada !28

En 1928, donc la même année que son essai sur le snobisme, Werfel publia sa grande nouvelle Der Abituriententag ${ }^{29}$ (La Réunion de promotion) - énorme succès de vente, 10000 exemplaires en une semaine ! - où émergent les conséquences criminelles du snobisme. Le récit se situe en 1902 et met en scène des potaches d'un lycée allemand de Prague. Ils sont en révolte contre les humanités classiques que veulent leur inculquer leurs maitres. Ils se piquent de poésie révolutionnaire et de littérature européenne contemporaine. Ils se livrent à l'occultisme, à l'alcool, à la sexualité. Leurs expérimentations linguistiques consistent en des inversions de proverbes ; prenant comme exemple le proverbe allemand « Tel qui creuse une fosse pour autrui y tombera lui-même » («Wer andern eine Grube gräbt, fällt selbst hinein »), ils le retournent en : "Tel qui tombe lui-même, creuse une fosse pour autrui » («Wer selbst hineinfällt, gräbt andern eine Grube »). Or cela est lourd de sens. En effet, ils entraînent dans leur débauche un élève juif spécialement doué ; celui-ci cède à leurs incitations, s'anéantit moralement et intellectuellement ${ }^{30}$. Mêlé à leurs fraudes sur les notes, il s'enfuit de 
Prague et nul ne sait ce qu'il advient de lui. Vingt ans plus tard, on soupçonne un moment qu'il ait pu commettre un assassinat. Le jeu idiomatique a porté ses fruits vénéneux; en tombant, eux, dans les désordres, ils ont précipité leur victime dans l'abîme. Dans Der Abituriententag le milieu scolaire pragois est fortement marqué comme autobiographique. Il semble que Werfel rappelle là une expérience intellectuelle et (im-)morale de sa jeunesse, qu'il appellera plus tard " avant-gardiste » et que nous pouvons tout à fait considérer comme équivalente du snobisme.

21 Ici se manifeste la volonté de puissance présente en tout être humain. Car le principal instigateur des dérèglements ne supporte pas la supériorité intellectuelle d'un autre, juif de surcroît. Si une moralité peut se dégager de la nouvelle, elle est sur la droite ligne du message de Werfel depuis ses premiers recueils lyriques : seule l'humilité peut étouffer l'agressivité naturelle. Au rebours, le snobisme ne fait qu'aggraver l'ambition illégitime. Il prend la dimension d'une révolte contre Dieu. Pris de remords d'avoir voulu éliminer son camarade surdoué, le narrateur du Abituriententag doit reconnaître : «Aber Gott hatte ihn begnadet und nicht mich. " ${ }^{31}$

Franz Werfel pense et juge exclusivement dans la sphère de la création littéraire et artistique. Il ramène toutes les carences psychologiques, sociologiques, morales, aux distorsions infligées aux règles - aux « lois » (!) - de l'expression. Après la dénonciation dans l'essai de 1928, viendront les propositions positives de solutions d'ordre spiritualiste et religieux, avec les grands essais des années trente: Realismus und Innerlichkeit (1931), Können wir ohne Gottesglauben leben? (1932), Von der reinsten Glückseligkeit des Menschen (1938)et les Theologumena. ${ }^{32}$ (13-195) Cette dernière partie avait été à coup sûr préparée par l'auteur, mort en septembre 1945, à fin de publication. Tout porte à croire qu'une grande partie des aphorismes qui la composent datent des années trente. Ce que Werfel disait dès 1928 à propos des snobs se retrouve condensé dans des passages des Theologumena:

Der Snobismus ist eine geistige Spielart des Parricidums, des Elternmords (durch Verleugnung). Zugleich aber ist er, wie jede Äußerung des Satanismus, ein verderbter, ein in Fäulnis übergegangener Wert, nämlich der pervertierte Drang zum Höheren. ${ }^{33}$ (181)

Ich habe viele Arten des Hochmuts erlebt, an mir und an andern. Da ich aber in meiner Jugend eine Zeitlang selbst dazugehört habe, kann ich aus eigener Erfahrung bekennen, dass es keinen verzehrenderen, frecheren, höhnischeren, teufelsbessesseneren Hochmut gibt als den der avangardistischen Künstler und radikalen Intellektuellen, die von eitler Sucht bersten, tief und dunkel und schwierig zu sein und wehe zu tun. Unter dem amüsiert empörten Gelächter einiger Philister waren wir die unansehnlichen Vorheizer der Hölle, in der nun die Menschheit brät. ${ }^{34}$ (191)

Autrement dit, après avoir frayé la voie des massacreurs bolcheviques, les avantgardistes émules de Maïakowski ont aussi fait le jeu des nazis. Il existe toutefois des cas de snobisme totalement indépendants de tendances culturelles. Que sont les auteurs du génocide arménien tels que présentés dans Die vierzig Tage des Musa Dagh (1933), si ce ne sont des snobs? Ils soignent leur physique, sont sanglés dans des uniformes élégants, assument cyniquement leurs crimes, imitateurs radicaux qu'ils veulent être des nationalistes européens du début du XIX ${ }^{e}$ siècle.

Comme dans Der Abituriententag Werfel lui-même bat sa coulpe. Or même si l'expressionnisme fait partie des avant-gardes, on ne peut pas dire que les productions de Werfel que l'on connaît de cette époque versent dans le grotesque ni l'ésotérisme, l'expérimentation radicale ni la provocation vulgaire. Il fit sensation par la nouveauté 
des rythmes, et surtout des images; mais, pour une bonne moitié, ses poèmes sont fidèles à la scansion classique, à la strophe, à la rime. Toutefois, en partant pour le front en 1914, Werfel écrivait à son éditeur Kurt Wolff qu'il laissait un tas de manuscrits "sans valeur " dans ses appartements de Leipzig et de Prague, et chez ses amis Ernst Pollack, Willy Haas, Otto Pick et Stefan Zweig. ${ }^{35}$ Aurait-il commencé, fort jeune encore, en donnant dans certains excès, dont les traces auraient disparu ?

Toujours est-il que l'essai sur le snobisme marque indubitablement l'adhésion définitive de Werfel au conservatisme tant en arts qu'en politique. "Der Plebejer, der die höhere Klasse zu erobern träumt! Dies die Urformel des Snobismus! » ${ }^{36}(266)$ Et de remarquer que le snobisme est fils des révolutions :

Ist es nicht bemerkenswert, daß nicht etwa zu Zeiten der scharf geschiedenen Kasten der Snobismus kulminiert, sondern zur Zeit der Revolutionen, der sich auflösenden Klassenordnung? Die Geburt des Wortes ,Snob' fällt in die Epoche der Cromwell-Revolution ${ }^{37}$ (269).

Là encore l'anarchisme antibourgeois est porté par une certaine littérature, celle des cafés et de la bohème : "alles was unter den Begriff des Kaffeehauses und der Bohème fällt $»^{38}$ (272). Aussi l'essai contre le snobisme sera-t-il suivi immédiatement, en 1929, par le roman Barbara oder Die Frömmigkeit (Barbara ou La piété), qui porte condamnation de la violence révolutionnaire et met en cause les littérateurs des cafés viennois. Sans doute Werfel eût-il repris à son compte l'indignation du critique et essayiste conservateur français Paul de Saint-Victor au moment de la Commune: "L'abîme appelle l'abîme, le blasphème intellectuel appelle le forfait social. Sous l'énergumène couvait l'incendiaire. $»^{39}$

Un essai n'est pas seulement intéressant pour le repérage des positions de l'auteur et la structuration de son parcours. Sa valeur d'appel direct, son avalanche d'exemples sont provocateurs et ne peuvent manquer de soulever bien des discussions. Classer Torquemada comme snob n'est-ce pas banaliser ses cruautés? Peut-on affirmer avec Werfel que, par rapport aux snobs modernes, Monsieur Jourdain est «malheureux et inoffensif » (" unglücklich und harmlos ») (269) ? Est-ce que le snob Napoléon détestait ses origines? Werfel ne va pas jusque-là, ce qui serait pourtant dans la logique de ses démonstrations. Or depuis, les archives ont parlé et révèlent les propos haineux de Napoléon contre la Corse et ses habitants ${ }^{40}$. Faut-il attribuer au snobisme l'adoption par Henri Beyle d'un nom de plume allemand emprunté à la ville natale de Johannes Winckelmann, ce que Werfel n'évoque pas? Les oppositions mentionnées plus haut «Konsonanz/Dissonanz », « Tonalität/Atonalität », menant à la définition du snobisme comme "atonalité de la mise en valeur de soi », impliquent-elles un discrédit de la musique moderne? Que non pas, l'atonalité n'est récusée qu'en tant qu'elle affecte la personnalité, et non comme ressource musicale, puisque dans Verdi, Werfel fait dire au maestro :

Nicht nur die Konsonanz, auch die Dissonanz ist ein vollkommen gleichberechtigtes

Mittel der Musik. Es ist vielleicht nur Faulheit, daß wir bloß eine gewisse Anzahl

harmonischer Verbindungen als Wohllaut gelten lassen ${ }^{41}$.

Plus importante est la question de fond : Werfel est dans le vrai en dénonçant l'amusement d'une partie de la société face à l'incohérence et à la violence. Mais une démarche de mise en valeur individuelle peut-elle prendre la dimension d'un phénomène collectif nuisible? Le terme de snobisme étant d'emblée adopté dans un sens dépréciatif, est-il crédible de l'identifier à un courant "satanique "? Et surtout : les innovations, voire provocations en art peuvent-elles être globalement taxées de 
snobisme, donc d'inauthenticité ? Werfel refuse d'accéder aux ressorts d'inspiration des créateurs qu'il récuse. Pour lui, leurs productions ne sont que prétentieuses excentricités et fuite devant l'effort qu'exige une créativité intelligible.

Aux lecteurs des essais en général de compléter ou de reconstruire selon leur propre logique ce qu'affirment les essayistes. Le grand sujet de réflexion est ici de savoir si le lien existe entre les audaces de l'art futuriste authentique et les nuisances sociétales. Confronté aux thèses de Werfel, on pourrait conjecturer : le radicalisme est toujours néfaste. En art, il fait fi du public et répand l'impression que tout est permis. De plus, si la provocation repousse toute limite sans se heurter aux interdits, elle perd sa signification. Ne rencontrant aucune réaction, elle frappe dans le vide ; plus de repères, ce qui ne peut que trouver ses prolongements sur le plan des mœurs et de la politique. Par contrecoup, une autre partie de l'opinion, indignée par les débordements, appelle la censure et la répression, ou du moins s'en accommode. Les amuseurs deviennent responsables de l'instauration de dictatures.

Nous nous retrouvons dans l'atmosphère de l'entre-deux-guerres en Allemagne. Une tendance en art et en littérature effectuait un retour à l'objectivité. Le renouvellement n'était plus coupure d'avec la réalité. C'était la «Neue Sachlichkeit» de la peinture et de la narration. Là, Werfel est très évidemment partie prenante. Parallèlement œuvraient les milieux artistiques qui pratiquaient un futurisme permanent. Le mouvement marquant était le dadaïsme, équivalent du surréalisme français, tandis que le journalisme politique profitait de la démocratie pour perdre toute mesure. C'est alors qu'un Werfel, qui avait lui-même vécu l'impuissance des poètes de la génération expressionniste à conjurer la Guerre Mondiale, se mit à prêcher la modération, en un mot le réalisme.

31 L'intolérance de Werfel est celle d'un prophète. Le commandement de ne pas se positionner au-dessus de la réalité, en snob, en romantique, en "sur-réaliste " constitue un avertissement contre un autre réalisme qui, lui, ne sera pas prôné, mais imposé : le réalisme socialiste dicté une fois pour toutes par le congrès des écrivains soviétiques en 1934, et la mise au pas - «Gleichschaltung " - de la littérature en Allemagne, assortie de l'élimination de "l'art dégénéré » et de la promotion d'un réalisme du sang et du sol : «Blut und Boden ».

La Neue Sachlichkeit, qui ne sacrifiait aucunement l'originalité, ne trouva évidemment pas grâce, elle non plus, sous les régimes totalitaires. Notoires ou moins célèbres, juifs ou non, les auteurs qui représentaient la Nouvelle Objectivité furent soumis aux mêmes persécutions et autodafés que les plus radicaux parmi artistes et publicistes. Pas plus que les expressionnistes, ni les uns ni les autres ne purent empêcher la guerre.

Werfel, Juif allemand de Prague, devenu viennois, puis fuyant en France et aux USA, se sentait « sans peuple ni pays ». Au lieu de prendre la pose en revendiquant une identité fallacieuse, il a médité sa situation, et adapté sa créativité :

Ihr Völker der Erde, mich rührt

Das Bleibende, das ihr vollführt.

Ich selbst, ohne Volk ohne Land

Stütz nun meine Stirn in die Hand. ${ }^{42}$

En exil, Werfel eut le mérite de ne pas se poser en donneur de leçons. Il se tint éloigné des polémiques qui divisèrent les proscrits. Ses essais, il les consacra à ses amis morts ou vivants, à l'identité intangible de l'Autriche annexée par Hitler, au destin d'Israël, au 
ressourcement spirituel. L'heure n'était plus aux discussions esthétiques sur l'enracinement ou l'émancipation de l'art.

\section{NOTES}

1. Der Snobismus als geistige Weltmacht, in : Jahrbuch des Paul Zsolnay Verlags, Vienne 1928, p. 9-34. Devait constituer un chapitre d'un livre intitulé Die Krisis der Ideale, qui ne vit pas le jour. Une version abrégée de l'essai paru dans Die neue Zeitung, Vienne, 25 janvier 1946. Version intégrale dans Franz Werfel: Zwischen Oben und Unten. Aufsätze. Aphorismen. Tagebücher. Literarische Beiträge, Munich, 1975, p.261-278. Dans la suite du texte, les numéros de pages entre parenthèses renvoient à cette édition.

2. « Le crime est élevé à la dignité d'un sport».

3. «... l'idéal anglo-américain dominant, idéal rationnel et cinétique, qui de surcroît dans nos pays est vidé de toute morale."

4. William Makepeace Thackeray (1811-1863), The Book of Snobs 1846-1847, prend appui sur la signification de « snob » comme savetier, autrement dit l'admiration pour des choses vulgaires.

5. « On trouve le snobisme [...] en tout homme sous la forme d'un foyer d'infection potentiel».

6. « Le snobisme est la volonté de se faire valoir atteinte de maladie».

7. « Le snob épris d'aristocratie, de cléricalisme, de vie artistique, de célébrité, est l'espèce la plus courante et inoffensive de nos campagnes ».

8. "Quand je lis une quelconque expression d'un fanatisme sanguinaire, je pourrais jurer que son auteur est un atrabilaire névrotique du niveau de culture de l'enseignement secondaire, qui, Torquemada raté, s'affaire à effacer les traces de sa vraie nature. La couleur de son parti ou de sa classe sociale est en l'occurrence parfaitement indifférente dans le cadre de notre étude. Car bien plus nombreux que le snob communiste surgit de toute part le snob nationaliste. Lui aussi prouve par ce radicalisme suspect son illégitimité, l'inauthenticité de son identification. »

9.

«Ton âme : vapeur, air comprimé, électricité !

Aux distributeurs d'aumônes, aux nombrilistes,

Que la hache danse sur leur calvitie!

À mort, à mort sous les coups! »

10. Marie-Claire Méry : Rudolf Kassner (1873-1959) : Un écrivain hors du temps ? Variations sur le thème de l'essai, in: Le Texte et l'idée, Centre de Recherches Germaniques et Scandinaves de l'Université Nancy 2, n 17, 2002, p. 167-190, ici p. 174-175.

11. Le fait/le théâtre, la possession/le geste, la position/le rôle, le pouvoir effectif/la prétention, le héros/l'acteur, l'objet/l'éclat, la réalité/les insignes, la loi/la transgression.

12. « Il existe une atonalité de l'instinct de se faire valoir ! C'est ça le snobisme. »

13. «La volonté de puissance! Qu'on écoute soigneusement le son de ce concept. Personne ne peut nier sa réalité. Mais cela ne résonne pas simplement comme un son, mais comme un accord, où plein de choses se retrouvent."

14. «... est-il vrai que l'homme rêve du pouvoir effectif, des épreuves pour le conquérir, des responsabilités, des dangers, des amères expériences qu'il faut y mettre pour s'y maintenir?» 
15. «On peut considérer l'imagination de sa propre valeur comme malade à partir du moment où elle s'éloigne en fonction contradictoire de la situation individuelle et sociale de la personne qui fantasme ».

16. Voir M.-C. Méry, ibid. p. 171.

17. «Le Verdi de Werfel relève déjà de l'essai dans son sujet même (réalisé sur un mode antithétique) » (i.e. sur la base de l'antithèse Verdi/Wagner) Ludwig Rohner Der deutsche Essay, Neuwied/Berlin, 1966, p. 574.

18. «[les] desperados de l'esprit de toutes nations, [les] compliqués et romantiques, dont Bayreuth devint le point de ralliement international ». Franz Werfel : Verdi. Roman der Oper (1923) Francfort/M. 1966, p. 87 (chap. 3/V)

19. Ibid., p. 26-27.

20. Ibid., p. 196.

21. "Le romantique était pour le dépassement de la réalité. »

22. «... le déguisement déjà, l'obsession de parader en costume historique, cette façon coquette d'afficher un passé glorieux, révèle un snob et un romantique, l'un des premiers snobs qui nous soient connus historiquement. »

23. "J'ai une horreur de notre sang. Sais-tu ce que je ferais de nous, si j'étais César ?! » Franz Werfel : Paulus unter den Juden, in : Die Dramen, Francfort/M., 1959, t. I, p. 476.

24. «De même il existe par centaines des Juifs, certes pas précisément des Torquemada, qui sont pris d'un bienheureux accès de faiblesse en respirant de l'encens, et d'un accès de faiblesse malheureux quand ils sont reconnus pour ce qu'ils sont. »

25. "C'est la haine de soi à l'état passionnel. » Otto Weininger, né en 1880, se suicida en 1903, à cause du peu de succès de son œuvre principale Geschlecht und Charakter. En 1907 le poète Rappoport édita son œuvre posthume et ses fragments sous le titre Die letzten Dinge. C'est ce livre que cite Werfel. En 1919, Anton Gerber publia le reste des aphorismes de Weininger ainsi que quelques lettres. Weininger est antiféministe et antisémite, bien qu'il soit juif. Dans Geschlecht und Charakter, il considère que la femme est livrée à l'instinct, tandis que l'homme a le privilège de l'esprit. Weininger attribue aux Juifs des traits de caractère essentiellement féminins. Dans son fragment autobiographique Ergu $\beta$ und Beichte, de 1920, Werfel évoque juste, sans la réfuter, la comparaison que fait Weininger entre les Juifs et les mouches! (p. 693)

26. "Vous n'êtes pas seulement le fils de votre peuple, mais avant tout le fils de l'assimilation, cette obscure contrainte qui pousse à effacer ou à couvrir d'opprobre toutes traces qu'on laisse derrière soi. "

27. Karl Kraus poursuivit ses attaques ad infinitum, jusqu'à sa mort en 1936.

28. Fackel no 759-765, p. 105-110.

29. Traduction française commercialisée: «Le passé ressuscité» Paris, 1930, 1986, 1992. (Traduction en de nombreux endroits très imprécise!)

30. La victime accepte, entre autres dépravations, d'accompagner ses « amis » dans un bar, où ils le jettent, à leurs frais, dans les bras d'une prostituée. Puis ils viennent observer les réactions du pauvre naïf en cette conjoncture ; celui-ci, humilié, est saisi d'une crise nerveuse (chap. 5).

31. «Mais c'est lui que Dieu avait comblé de dons, et non pas moi." Franz Werfel: Der Abituriententag, Francfort/M. 1990, p. 87 (chap. 4).

32. Réalisme et intériorité, Pouvons-nous vivre sans foi en Dieu?, De la plus pure félicité de l'homme. Ces écrits furent regroupés dans le premier recueil intitulé Zwischen Oben und Unten, Francfort/M., 1946.

33. «Le snobisme est une nuance intellectuelle du parricide, de l'assassinat de ses parents (en les reniant). Mais il est en même temps, comme toute expression du satanisme, une valeur pervertie, tombée dans le pourrissement, à savoir la perversion de ce qui nous pousse vers le haut. »

34. "J'ai connu maintes formes d'orgueil, chez moi et chez d'autres. Mais puisque pendant un certain temps, dans ma jeunesse, j'en ai fait moi-même partie, c'est sur la base de ma propre 
expérience que je peux confesser qu'il n'est pas d'orgueil plus dévorant, plus arrogant, plus insultant, d'une pire possession diabolique que celui des artistes avant-gardistes et intellectuels radicaux, emplis jusqu'à en éclater de la vanité maniaque de se montrer profonds, et obscurs, et difficiles d'accès, et de faire mal. Sous les rires goguenards et faussement indignés de quelques béotiens, nous étions les minables en charge du préchauffage de l'enfer dans lequel l'humanité est en train de rôtir. "

35. Kurt Wolff : Briefwechsel eines Verlegers 1911-1963, hg. von Bernhard Zeller und Ellen Otten (1966), Frankfurt/M. 1980, p. 105.

36. «Le plébéien qui rêve de conquérir la classe supérieure ! La voilà la formule originelle du snobisme!»

37. « N'est-il pas remarquable que ce n'est pas aux époques où les castes sont nettement séparées que le snobisme culmine, mais au temps des révolutions, de la dissolution de l'ordre des classes sociales ? La naissance du mot "snob" tombe à l'époque de la révolution de Cromwell. » (On pense aux « incroyables » et « merveilleuses » sous la Convention thermidorienne et le Directoire !)

38. «Tout ce qui tombe sous la dénomination des cafés littéraires et de la bohème. »

39. À propos de Jules Vallès, d'abord écrivain bohème, conspuant les classiques, pamphlétaire déchaîné, puis communard actif. In : Paul de Saint-Victor, Barbares et bandits. La Prusse et la Commune, recueil d'articles, Paris, 1872, p. 246 (article «L'orgie rouge », 13 juin 1871, p. 241-255).

40. Voir Henri Guillemin, Napoléon tel quel, Paris 1969.

41. «Non seulement la consonance, la dissonance elle aussi est une ressource de la musique à pleine égalité. Peut-être n'est-ce que paresse si nous n'admettons comme mélodieuses qu'un certain nombre de combinaisons harmoniques ». Verdi (note 18) p. 342 (chap. 9).

42. «Vous, peuples de la terre, m'émeuvent / Les permanences que vous réalisez. / Pour moi, sans peuple ni pays / Me voilà, le front appuyé sur ma main ». Das Bleibende in Franz Werfel : Das lyrische Werk, hg. von Adolf Klarmann, Frankfurt/M. 1967, p. 432 (recueil Schlaf und Erwachen [« Sommeil et réveil »], 1935).

\section{INDEX}

Mots-clés : Neue Sachlichkeit, Nouvelle objectivité

oeuvrecitee Der Abituriententag, Der Snobismus als geistige Weltmacht, Die vierzig Tage des Musa Dagh, Verdi. Roman der Oper

\section{AUTEURS}

\section{MICHEL REFFET}

Université de Bourgogne 